\title{
Painel de visualização de dados para gestão federal do Serviço de Atendimento Móvel de Urgência (SAMU)
}

\section{Dashboard for federal management of the Mobile Emergency Care Service (SAMU)}

Leandro Manuel Reis Velloso, Lucia Vilela Leite Filgueiras, Mailson Fontes de Carvalho, Luiz Sérgio de Souza, Luciano Anísio Garcia, Johne Marcus Jaske, Tania Letícia dos Santos, Gabriela Momberg Araújo, Henrique Mathias Fernandes, Solange Alves-Souza, Marcia Ito, Átila Szczecinsky Rodrigues

infovis, SAMU, codesign

A gestão da Saúde Pública no Brasil é carente de recursos para a visualização de informações. Como parte de pesquisa para a melhoria dos painéis apresentados aos gestores, este artigo apresenta o protótipo funcional de um painel de visualização de dados criado para auxiliar a gestão e a operação do Serviço de Atendimento Móvel de Urgência (SAMU) em nível federal. Os métodos de pesquisa e intervenção adotados na criação e no desenvolvimento do artefato são pautados pela Design Research e PesquisaAção. Abordagens centradas no usuário guiaram as etapas de trabalho da equipe interdisciplinar e o envolvimento cocriativo dos gestores do SAMU. Como resultado deste esforço, detalham-se as soluções de arquitetura e design da informação, como a organização dos painéis por escopo territorial, o uso de séries temporais, mapas, componentes interativos e pictogramas para apresentação das unidades móveis. É descrito o modo como o artefato organiza a complexidade da rede de atendimento fornecendo visões por território, central de regulação, base descentralizada e unidade de atendimento. São apresentados ainda resultados compilados como requisitos de projeto para melhorias futuras. A partir da avaliação realizada com outros gestores, conclui-se que o protótipo apresentou melhorias no processo de operação do SAMU o protótipo apresentou melhorias no processo de operação do SAMU.

infovis, SAMU, co-design

Public health management in Brazil lacks information visualization resources. As part of a research project intending to improve dashboards presented to public health managers, this article presents a functional prototype of a dashboard designed to support the management and operation of the Mobile Emergency Care Service (SAMU) at the federal level. The research and intervention methods adopted during the creation and development of the artefact are based on Design Research and Action Research. Usercentred approaches have guided the interdisciplinary team's work stages, as well as the co-creational involvement of SAMU managers. The information architecture and design solutions are specified, such as the arrangement of panels by territorial scope, or the use of time series, maps, interactive components and pictograms to present mobile units. The article depicts how the complexity of the care network is organized by the dashboard, which provides views by territory, regulation central, decentralised base and care unit. Results compiled as design requirements for future improvements are also presented. Based on evaluation sessions with other managers, we conclude that the prototype has brought improvements to the SAMU operation activities. Curitiba | Brazil | 2021 


\section{Introdução}

A ideia de que o principal objetivo da visualização da informação (infovis) é permitir aos olhos e ao cérebro perceber o que está além de seu alcance, está presente em grande parte da literatura especializada (Cairo, 2013). Esta característica reveladora da infovis indica seu grande potencial para apoiar a tomada de decisão em áreas como a saúde pública, especialmente em um país com as dimensões do Brasil (Tamaki et al., 2012). Ao mesmo tempo, a transparência dos dados, tema de interesse público, demanda um trabalho de publicização da informação, na qual a visualização de dados também pode desempenhar um papel determinante (Schoffelen et al, 2015).

A saúde é considerada um direito de todos os cidadãos brasileiros, conforme estabelece a Constituição Federal de 1988 (Constituição, 1988, art. 196). Como concretização desse direito, foi elaborada a lei n 8080 de 19/9/1990, que dispõe sobre a organização e funcionamento do Sistema Único de Saúde (SUS). A organização do SUS é essencialmente tripartite, sendo constituída por uma rede de serviços e ações gerida por níveis de governo federal, estadual e municipal de forma não-hierarquizada (Paim, 2009). Diante da complexidade deste sistema, são essenciais para sua manutenção as atividades de monitoramento e a avaliação. Com este objetivo, foi instituída pelo Ministério da Saúde a Sala de Apoio à Gestão Estratégica (SAGE), através da portaria $n^{\circ} 406$ de 8 de março de 2012 (Ministério da Saúde, 2012a), que tem a visualização de dados organizada em painéis, como sua principal contribuição.

Diante de uma demanda do Ministério da Saúde, o projeto Infovis para a Saúde Pública foi concebido com a proposta de aprimorar a experiência do usuário com a visualização da informação na SAGE. Com uma equipe formada por designers da informação, cientistas de dados, pesquisadores de saúde pública, técnicos e gestores públicos, estabeleceu uma abordagem interdisciplinar em torno de práticas colaborativas de pesquisa, criação e desenvolvimento de protótipos. O projeto, desenvolvido ao longo de 2020, foi financiado pela Organização Pan-Americana da Saúde (OPAS) e executado pela Fundação de Apoio à Universidade de São Paulo (FUSP) em parceria com o Ministério da Saúde (MS) e resultou em um conjunto de painéis que respondem a necessidades expressas pelos gestores públicos.

\section{Contexto}

O painel de visualização do SAMU foi um dos protótipos desenvolvidos na pesquisa Infovis para Saúde Pública, tendo em vista dar suporte para a gestão federal no monitoramento da rede e das unidades locais distribuídas no território nacional.

O principal objetivo do Serviço de Atendimento Móvel de Urgência (SAMU) é o atendimento às vítimas em situações urgentes, sejam de natureza clínica, obstétrica, traumática, pediátrica, psiquiátrica ou outra (Ministério da Saúde, 2012b). Trata-se de um programa federal com gestão descentralizada para os estados e municípios. Fica a cargo da gestão federal atender às demandas locais e monitorar suas unidades, como ambulâncias, motos, lanchas e aeronaves. 
A tarefa de referência deste painel é a visualização geográfica da cobertura, das Centrais de Regulação de Urgência (CRU), das bases e do status e detalhes das Unidades de Suporte Básico (USB).

Os requisitos definidos para criação do painel tiveram origem em centenas de desejos identificados em uma etapa de imersão, obtidos a partir de entrevistas semiestruturadas realizadas com usuários e especialistas, de dentro e de fora do Ministério da Saúde, além de revisões bibliográficas e da análise de benchmarks.

Os desejos selecionados foram analisados e convertidos em "histórias do usuário", de forma que o problema pudesse ser recombinado de modo exequível. A partir desta síntese os principais requisitos foram descritos da seguinte forma:

- Exibir visualização espacializada das $C R U$, bases descentralizadas e municípios, por cobertura do SAMU;

- Exibir listagem das CRU e das bases correspondentes ao território visualizado, com informações sobre profissionais, unidades e atendimento;

- Exibir o detalhamento de cada unidade móvel, com informações sobre o veículo e respectivas atividades;

- Exibir valores de financiamento por central, município, região e unidade federativa;

- Exibir a cobertura no território.

\section{Materiais e métodos}

O projeto teve como base teórica as abordagens da Design Research (Simonsen et al, 2010), que faz uso de protótipos e artefatos de design, acrescentando uma dimensão pragmática ao conhecimento teórico, o que a diferencia da pesquisa científica tradicional, e da Pesquisa-Ação (Thiollent, 2011), que fornece base para o envolvimentos dos usuários e dos agentes interessados em um processo colaborativo. Tanto a Design Research quanto a Pesquisa-Ação se constituem como métodos qualitativos que valorizam o aspecto científico da atividade coletiva e prática do design. De acordo com Thiollent (2011), diferentemente de modelos de consultoria, a Pesquisa-Ação promove uma cultura de compartilhamento de informações e conhecimento entre os pesquisadores e partes interessadas.

As etapas de projeto ocorreram seguindo as premissas do Lean UX (Gothelf \& Seiden, 2013), abordagem colaborativa, ágil e centrada na experiência do usuário. A centralidade e o envolvimento do usuário não ocorreu apenas durante a fase de levantamento de dados, mas durante toda a fase de criação e desenvolvimento do protótipo, aproximando o projeto das práticas do codesign (Sanders \& Stappers, 2008). O painel foi foco de atenção da equipe durante um período de três semanas em um sprint que envolvia reuniões diárias com especialistas como gestores do próprio SAMU em nível federal.

O ciclo que caracterizou o sprint ocorreu de modo inteiramente remoto tendo sido formado por uma semana de ideação, seguida de uma semana de prototipação em software gráfico, para ser concluído em uma semana dedicada à programação do protótipo funcional. Durante a 
primeira semana os designers trabalharam gerando alternativas através de sketches (Figura 1) em um quadro digital online ${ }^{1}$, que recebia, em reuniões diárias, comentários e anotações dos especialistas e usuários, fazendo uso de um método colaborativo definido por Van der Lugt (2002) como brainsketching, em referência ao brainstorming. Na segunda semana, os sketches já validados serviram de base para a prototipação de alta fidelidade realizada em softwares gráficos ${ }^{2}$, também usados para validação. Na terceira e última semana, foi entregue um protótipo funcional, com frontend desenvolvido (HTML, CSS e JS) e um modelo de integração com dados no formato JavaScript Object Notation (JSON), além de documentação em uma página web anexada ao painel, contendo uma ficha técnica sobre os dados e os gráficos e uma lista de melhorias (backlog).

Figura 1. Sketch resultante da primeira fase de ideação.

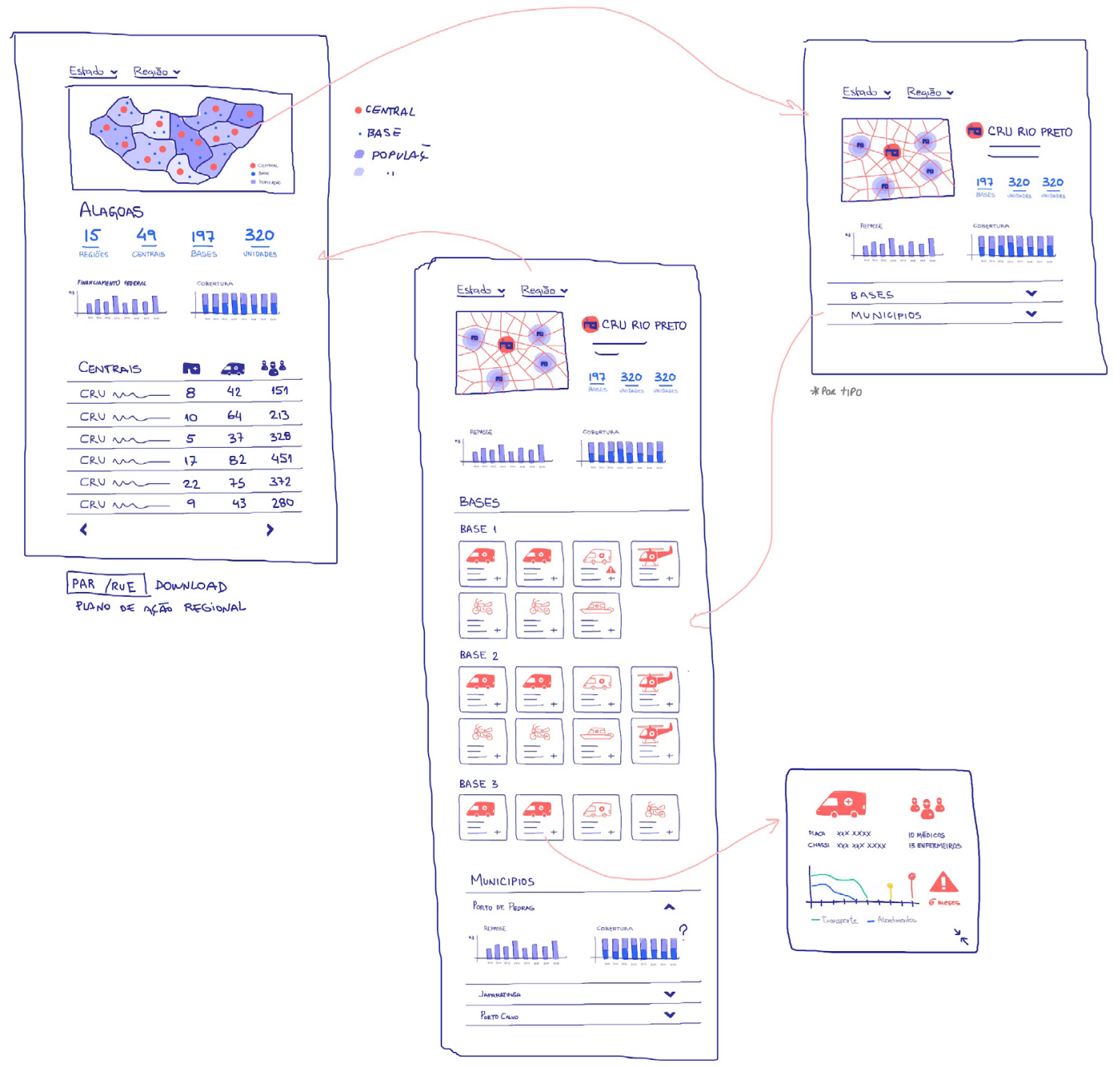

\footnotetext{
${ }^{1}$ http://miro.com

${ }^{2}$ http://figma.com
} 
No desenvolvimento dos gráficos do painel, foi utilizada a biblioteca Plotly.js ${ }^{3}$, constituída por um conjunto de gráficos personalizáveis pré-programados em linguagem Javascript. Para os mapas foi utilizada a tecnologia OpenStreetMap ${ }^{4}$, que fornece a base de dados para constituição de mapas georreferenciados, com apoio da biblioteca Leaflet ${ }^{5}$, que permite a personalização de padrões visuais para mapas interativos.

\section{Resultados}

O diagnóstico originado pelas dinâmicas de co-design indicou a predominância de dados categóricos e múltiplos níveis de hierarquia na estrutura do SAMU. A partir disso, foi elaborado um painel que consiste na representação da distribuição territorial das unidades de saúde para racionalização dos recursos e monitoramento de operações. É dividido em duas abas principais, sendo a primeira baseada em limites administrativos, e a segunda, em pontos correspondentes às CRU. Pode-se considerar quatro níveis de organização da informação nestas páginas (Figura 2): 1) Territorial; 2) Cobertura das centrais; 3) Agrupamento de unidades móveis por base descentralizada; 4) Detalhamento dos dados da unidade móvel.

Figura 2. Apresentação dos quatro níveis de organização da informação presentes no painel

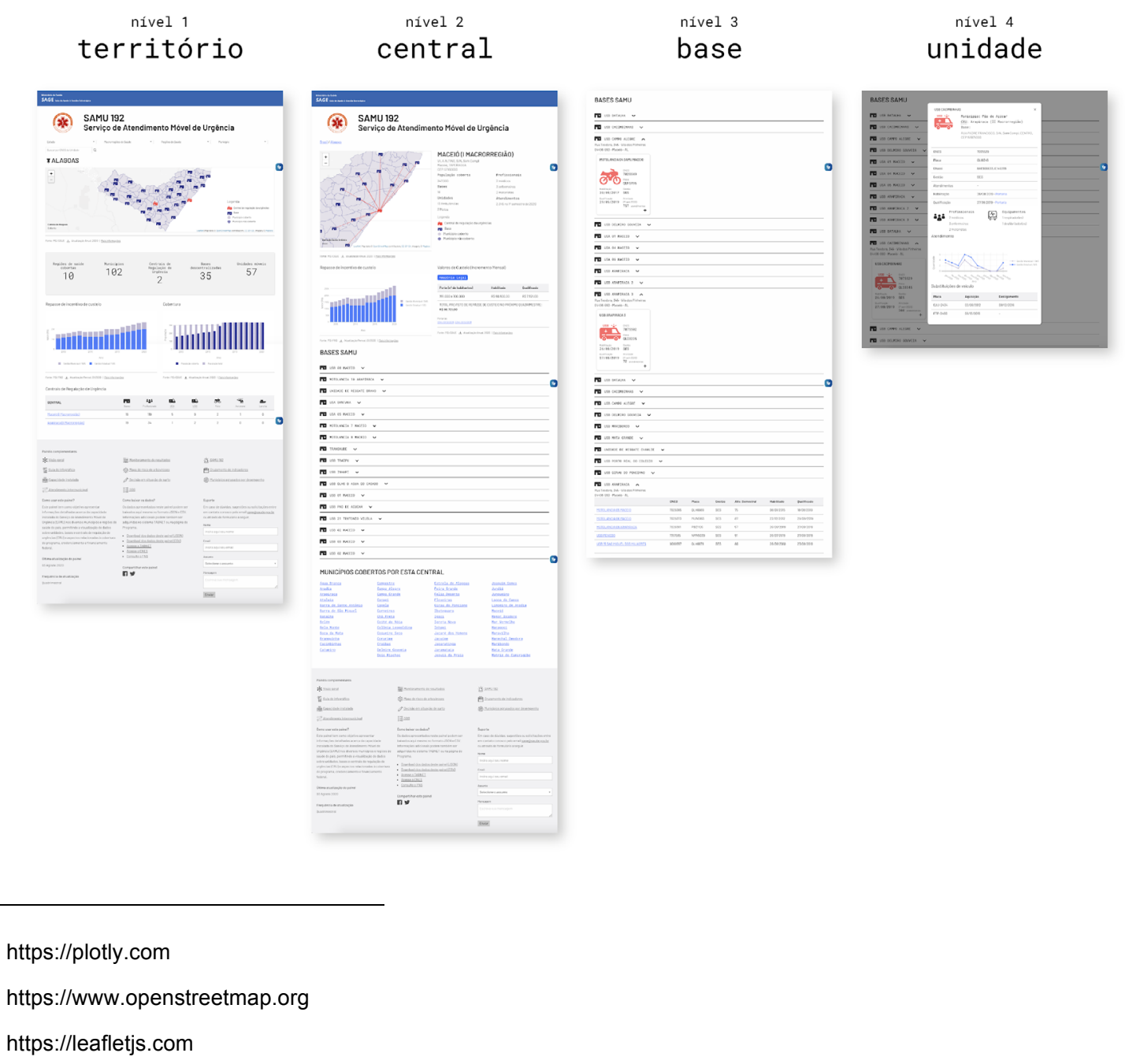


Uma das principais estratégias de comunicação dos dados foi o emprego de símbolos em mapas e gráficos, como destacado na Figura 3. Os pictogramas permitiram a visualização de informação categórica no painel, determinada por dados provenientes do Cadastro Nacional de Estabelecimentos de Saúde (CNES). Assim, pudemos caracterizar estabelecimentos como central ou como base, e unidades móveis sob as categorias Unidade de Suporte Básico (USB), Unidade de Suporte Avançado (USA), moto, lancha, ou aeronave. Além disso, foram utilizados pictogramas para identificar dados de equipe e equipamentos, bem como para indicar situações de risco. Este sistema de categorização foi implementado em diversos componentes gráficos do painel: mapa, tooltip, tabela, card e modal, com variações a depender da função de cada visualização (Figura 4). O manual de identidade visual do SAMU foi uma das referências ${ }^{6}$ para a composição do conjunto de pictogramas.

Figura 3. Destaque da presença de símbolos e pictogramas no painel

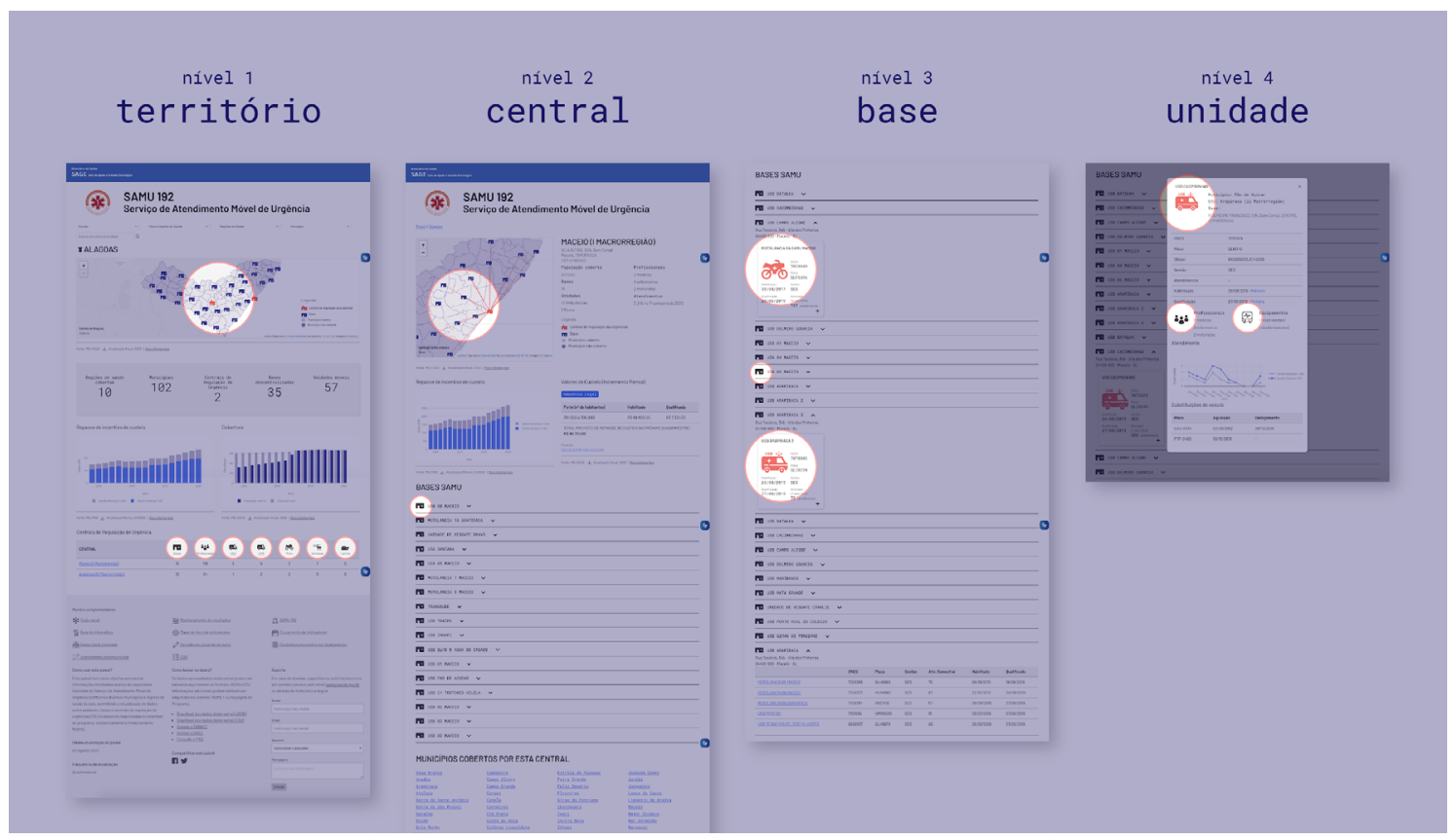

\footnotetext{
${ }^{6}$ Pictograma da USA modificado a partir de Sergey Demushkin. Pictograma do helicóptero criado por Tezar Tantular. Pictograma da motocicleta modificado a partir de "icon 54". Pictograma da lancha modificado a partir de Sebastian Ilves. http://thenounproject.com
} 
Figura 4. Sistema de pictogramas organizado segundo uso em elementos gráficos do painel.

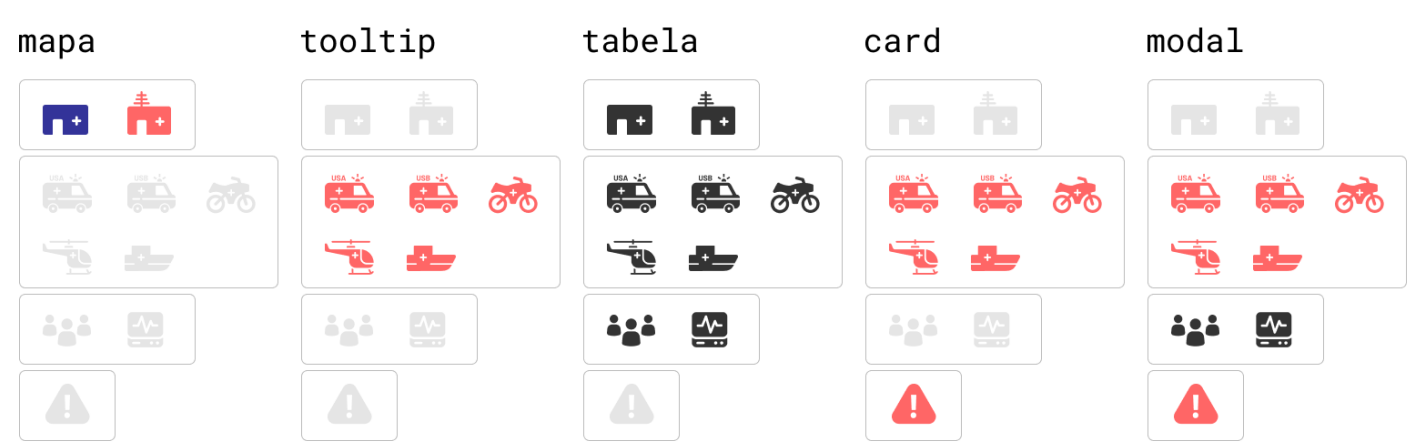

A visão baseada em limites territoriais (Figura 5) pode assumir as escalas federal, estadual e municipal, bem como de regiões e macrorregiões de saúde. Em todos estes casos, um mapa com duas variáveis é exibido, combinando a representação coroplética de municípios cobertos e descobertos, e uma camada de símbolos que indica localização de estabelecimentos, sejam CRU ou bases. A interação com os símbolos permite a visualização de detalhes sobre o estabelecimento, incluindo outros pictogramas para identificar dados de veículos disponíveis (Figura 6). Abaixo do mapa tem-se um bloco de big numbers que resume as informações territoriais, de cobertura e de estabelecimentos.

Figura 5. Apresentação do mapa em níveis estadual (Alagoas) e municipal (Marechal Deodoro).

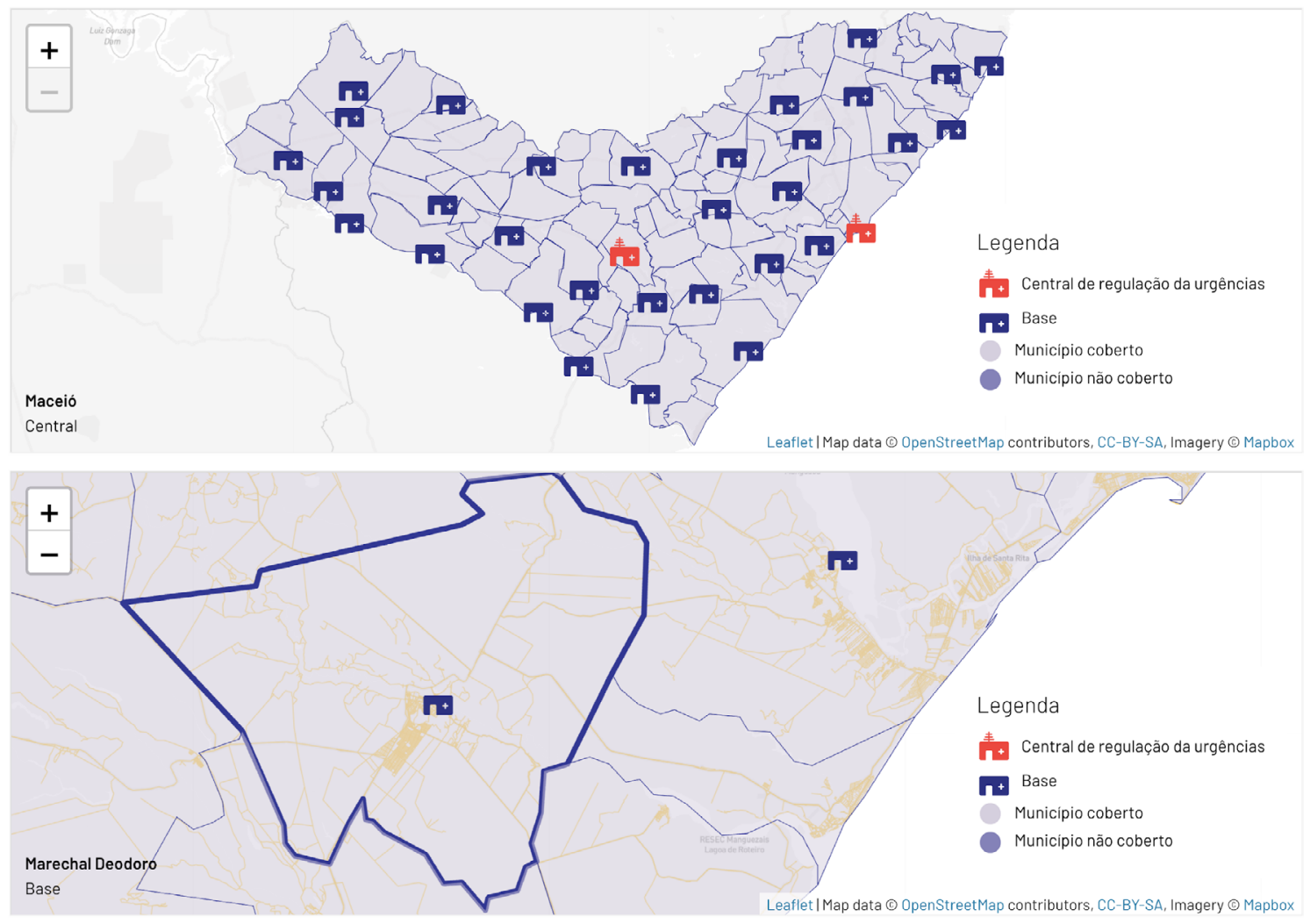


Figura 6. Apresentação de interações a partir do mapa: seleção de polígono, tooltip sobre centrais e tooltip sobre bases.

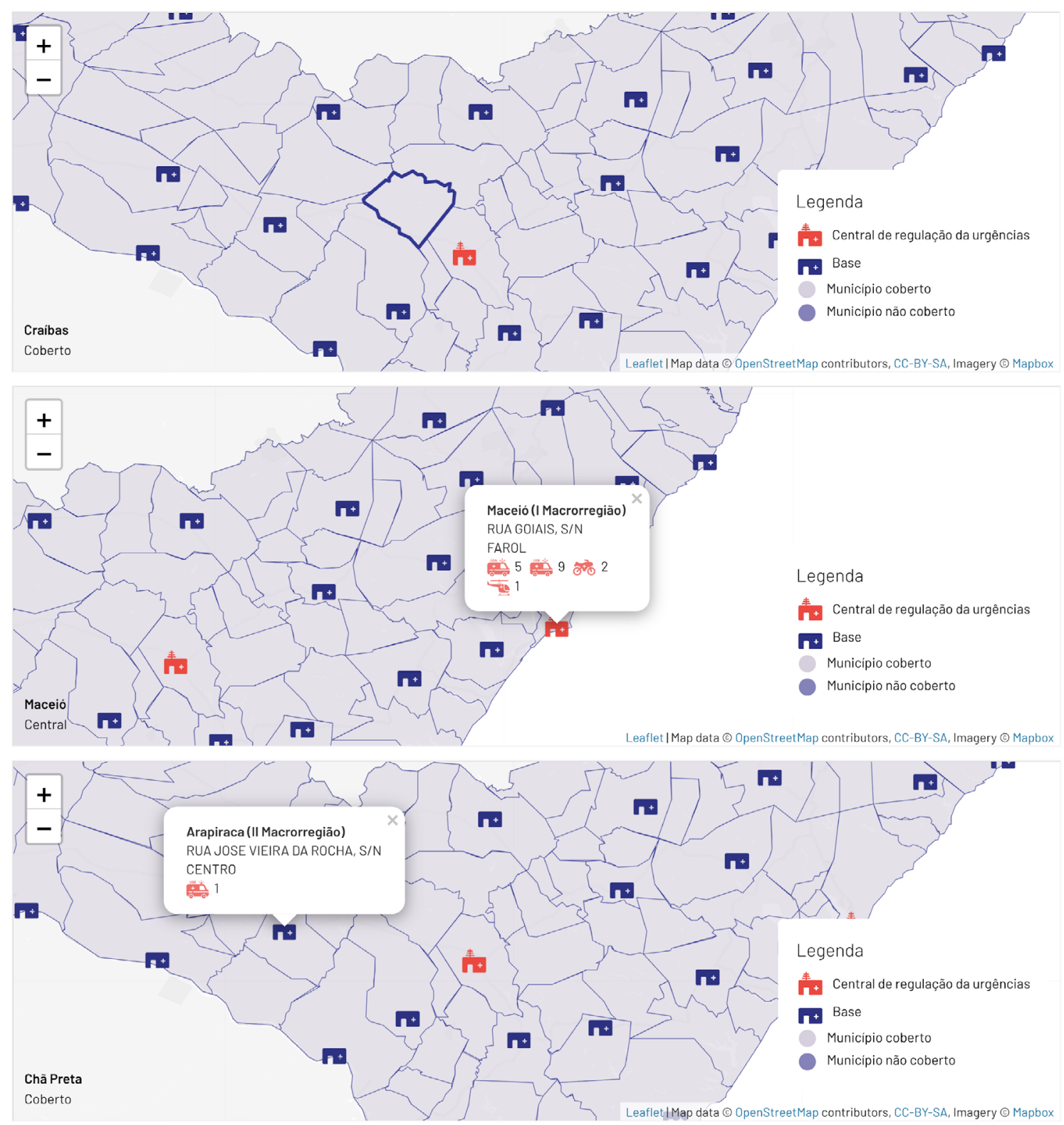

Em outra configuração, a visão de centrais organiza as informações relativas a uma CRU selecionada. O mapa exibe a região de abrangência da central a partir de três representações: coroplética, símbolos e linhas (Figura 7). Essa sobreposição de camadas é importante para o entendimento de que alguns municípios são cobertos mesmo que não possuam bases ou centrais em seu território. As linhas de conexão, por sua vez, representam a relação das bases com sua respectiva $\mathrm{CRU}$. Como não há dados sobre o tempo de deslocamento em função das características locais da malha viária, optou-se por apresentar as linhas de conexão sobre um mapa que exibe a malha viária. Desta forma, espera-se que o usuário conhecedor do território possa fazer a inferência quanto ao tempo de deslocamento. Ao lado do mapa consta um resumo de informações quantitativas da central: população coberta, bases, unidades, 
profissionais e atendimentos prestados no semestre. A página exibe, ainda, uma lista das bases como abas em dropdown.

Figura 7. Visualizações do mapa na aba das CRU. À esquerda, CRU de Arapiraca-AL; à direita, CRU de Maceió-AL.
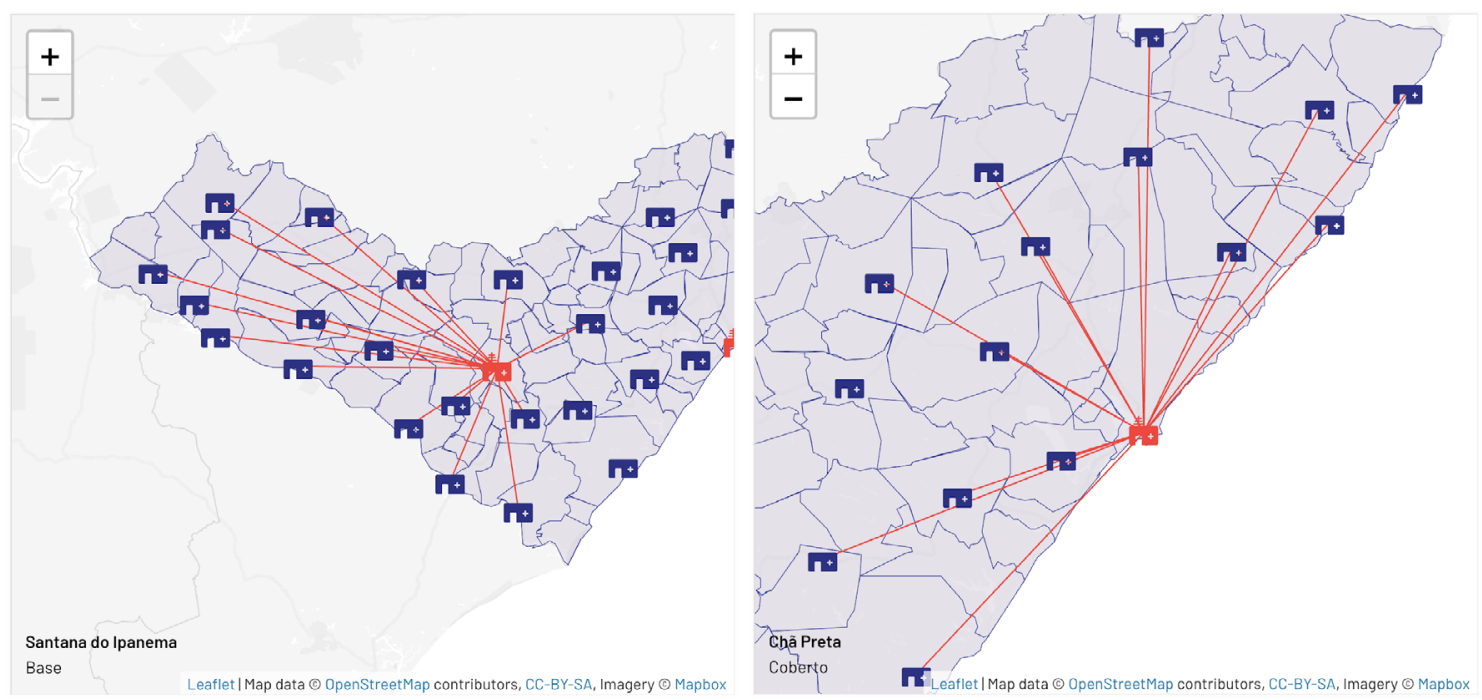

A visão das bases na página de central consiste em abas expansíveis, onde são agrupados os cards que representam cada unidade móvel presente na base. Havendo mais de quatro unidades, a visualização é substituída por uma tabela. Os cards apresentam um resumo de identificação da unidade móvel, incluindo o pictograma da categoria à qual pertence. A cor de fundo funciona como indicador do status de habilitação do veículo, e ícones de alerta ficam visíveis caso haja risco de desabilitação (Figura 8). Esta informação é de utilidade prática para o gestor, que pode verificar as condições de suspensão ou reabilitação de uma unidade móvel.

Figura 8. Tipologias de card segundo status: habilitado (esquerda); em risco por 2 meses sem atendimento (centro); em risco por 4 meses sem atendimento (direita).

\section{$n^{+}$Nome da Base}

Rua Rio Grande, 123 - Vila dos Campos 12345-000 - Município - UF

\begin{tabular}{|c|c|}
\hline \multicolumn{2}{|c|}{ NOME DA UNIDADE A } \\
\hline USA & \\
\hline+ & 0000000 \\
\hline 0 & $\frac{\text { Placa }}{X Y Z-0000}$ \\
\hline Habilitado em & Gestão \\
\hline DD/MM/AAA & SES \\
\hline Qualificado em & Atividade semestral \\
\hline DD/MM/AAA & 197 \\
\hline
\end{tabular}
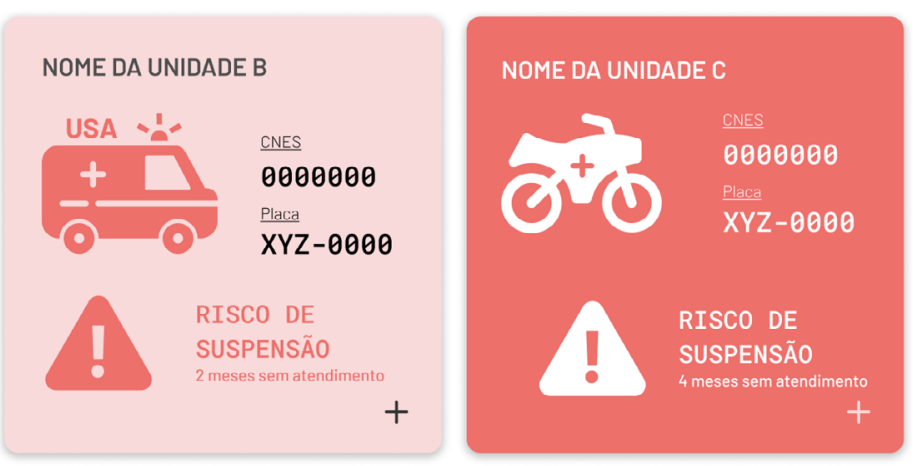
A interação com os cards de resumo permite a expansão do elemento em um modal (Figura 9). Com isso, tem-se a visualização de detalhamento da unidade móvel, na qual consta uma lista de equipamentos e profissionais, um gráfico de atendimentos e uma tabela com o histórico das substituições de veículos. A lista de equipamentos, assim como a de profissionais, apresenta as quantidades por categoria.

Figura 9. Visualização dos dados de uma Unidade Móvel por meio do elemento modal.

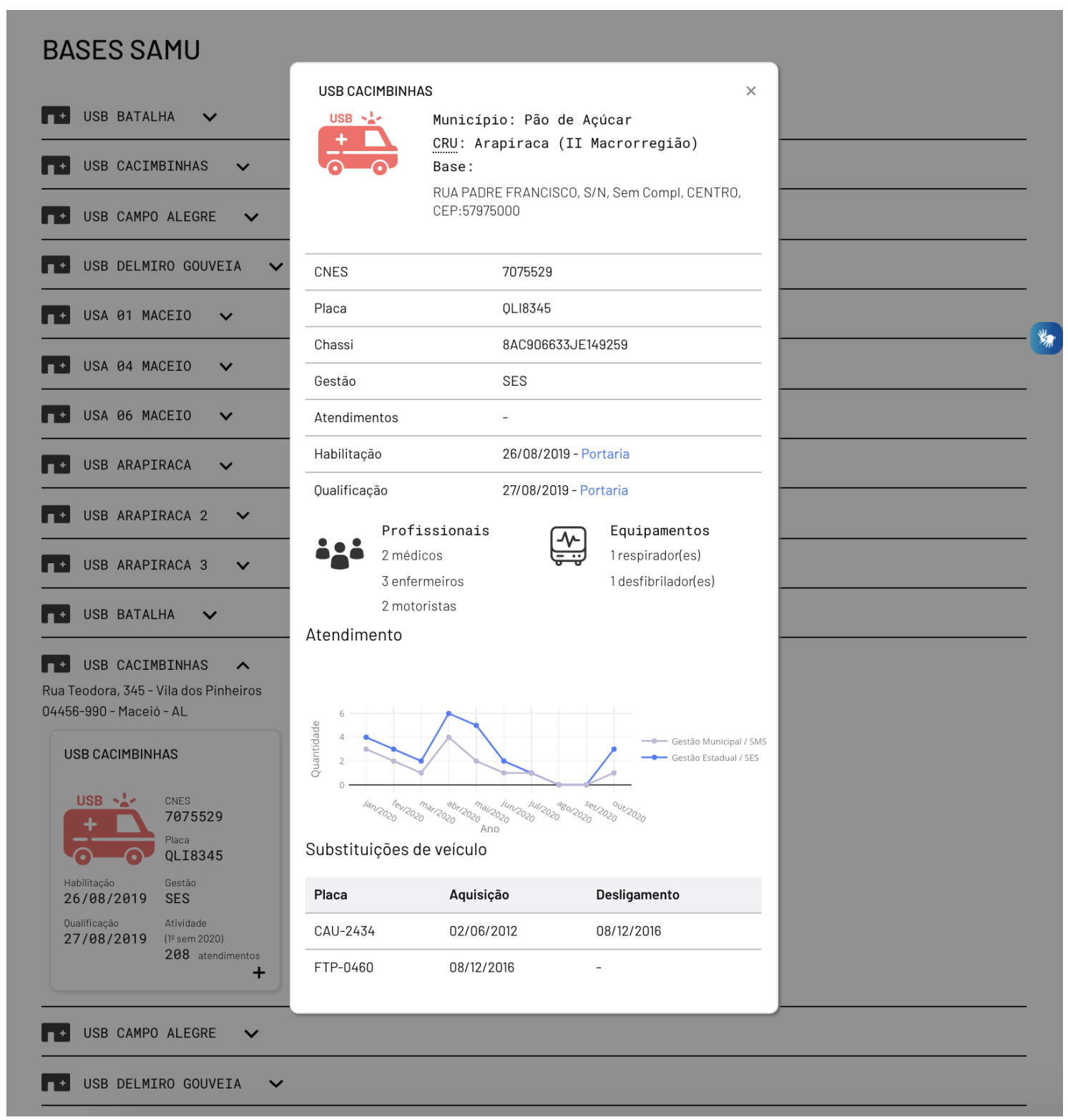

Tanto nas páginas de território como de central, há uma tabela ao final da página que lista os dados apresentados em mapa. O painel conta, ainda, com gráficos de série histórica do repasse financeiro e de cobertura do serviço, ambos com periodicidade anual. Nas visões de município e de CRU (central), os valores de custeio são apresentados em uma tabela. 
As visualizações do painel (Figura 10) são listadas a seguir:

1. Mapa com camada tripla exibindo estados, regiões e municípios cobertos, rede rodoviária e localização das bases descentralizadas e CRU.

2. Mapa exibindo municípios cobertos por uma CRU e suas bases descentralizadas.

3. Big Numbers exibindo $\mathrm{CRU}$, bases e unidades.

4. Diagrama com histórico do repasse por estado, município e CRU.

5. Diagrama com histórico da cobertura do SAMU por estado.

6. Tabela de custeio (incremento mensal) previsto por estado, município e CRU.

7. Tabela com lista de $\mathrm{CRU}$, profissionais e quantidade de bases e de unidades por tipo (USA, USB, moto, lancha e aeronave).

8. Lista das bases descentralizadas por CRU.

9. Cartões ou tabela com resumo dos dados de cada unidade.

10. Tabela detalhada dos dados da unidade.

11. Diagrama com série temporal dos atendimentos da unidade.

Figura 10. Esquema de organização da informação e respectivas visualizações, numeradas conforme a lista de visualizações descrita no texto.

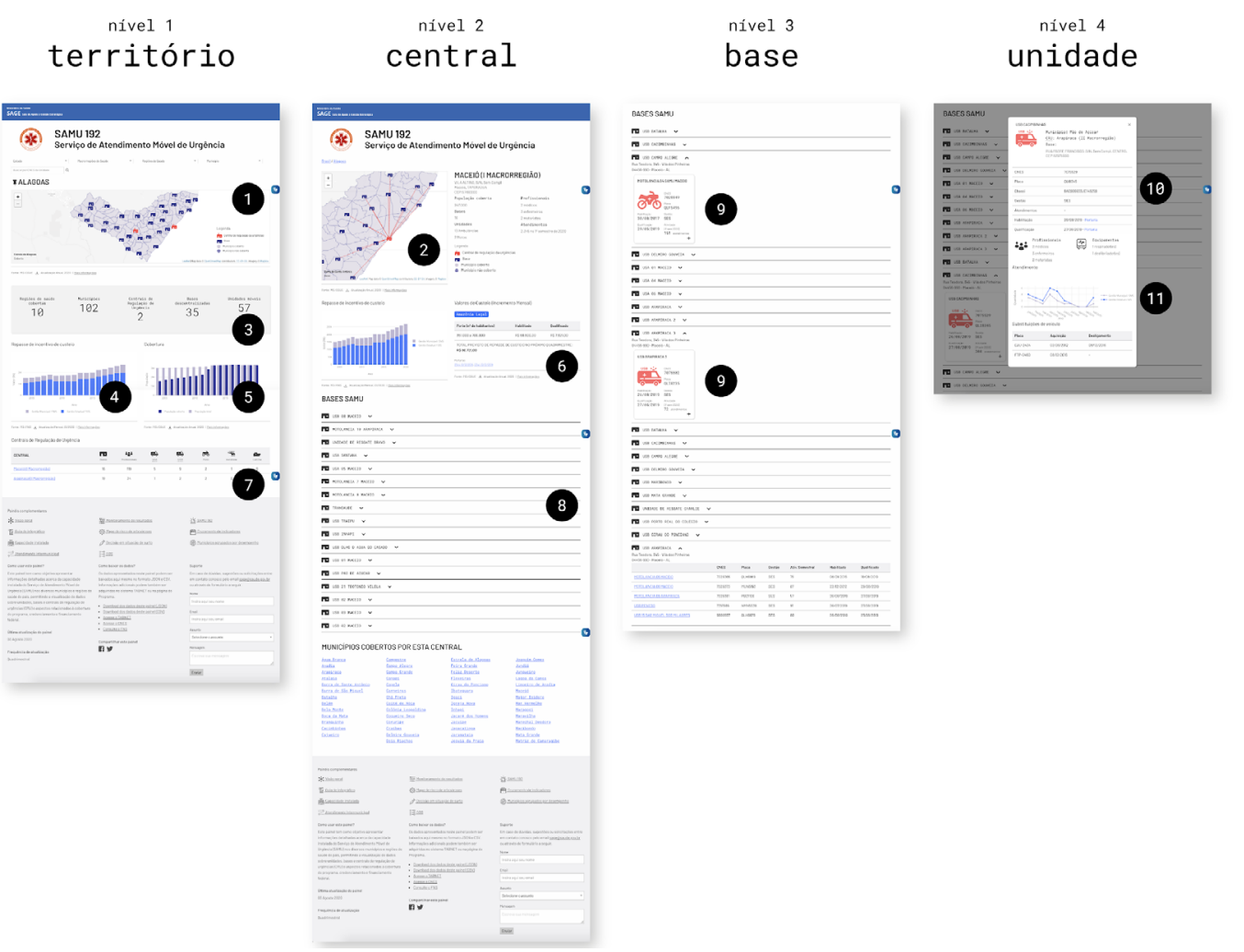

\section{Discussão}

Um dos aspectos marcantes do desenvolvimento deste painel foi a participação intensa dos usuários gestores do SAMU. Pode-se, portanto, considerar que a hierarquia da informação e os padrões de visualização propostos estejam intimamente ligados ao processo de tomada de 
decisão dos gestores federais, aos quais o painel se destina, o que define o público-alvo como organizacional (Sarikaya et al, 2019).

Em termos de propósito, o painel pode ser caracterizado como operacional, pois visa fornecer subsídios para a avaliação de operações do SAMU (Sarikaya et al, 2019). Uma vez que o painel foi elaborado com esta finalidade, seria fundamental que correspondesse à rotina de trabalho dos usuários gestores federais. O êxito do design, nesse caso, está em grande medida atrelado ao nível de integração com demais áreas relacionadas à cadeia de coleta e processamento dos dados.

Um dos desafios da gestão do SAMU é monitorar as unidades de saúde para racionalização dos recursos e devidas habilitações de veículos. O protótipo proposto oferece um modo de monitoramento de uso dos veículos no tempo, tanto com o uso de cores nos cards, que indica o risco de suspensão dos recursos após três meses de inatividade, quanto através de um gráfico de série temporal que mostra a quantidade de atendimentos mensais. A implementação deste gráfico no entanto é complexa, visto que a frequência de atualização dos dados das unidades é trimestral, dependendo portanto de outras fontes como dados dos hospitais sobre a entrada dos casos urgentes. Outro fator que aumenta esta complexidade são os chamados que não resultam em pronto atendimento hospitalar.

Apesar de não ter como foco a operação tática de apoio aos chamados, o painel também seria beneficiado com informações de georreferenciamento dos veículos. Este tipo de informação poderia indicar deslocamentos não previstos pelas CRU e até usos indevidos das unidades. O recurso potencializaria ainda outras soluções, como a identificação de locais com mais acidentes e áreas de risco, ou melhoria da logística dos atendimentos (Souza-Junior et al., 2016).

A priorização dos requisitos, na fase de análise dos dados, fez também por gerar um conjunto de "histórias do usuário" que compõem um backlog para possíveis melhorias a serem verificadas e implementadas em futuros ciclos de projeto. Dentre elas destacamos:

- Implementação de links para os Plano de Ação Regional (PAR) e a Rede de Atenção à Urgências e Emergências (RUE);

- Visualização do tempo de resposta do atendimento de cada unidade e de cada central;

- Visualização de unidades de atendimento que respondem aos chamados para a CRU mas que não possuem CNES;

- Informações sobre o custo operacional médio das unidades e bases;

- Cruzamento das bases com a rede de atendimento hospitalar;

- Visualização dos indicadores operacionais da CRU (ex.: chamadas abandonadas);

- Acesso aos links para as portarias de desabilitação de unidades.

\section{Conclusão}

O artigo apresentou resultados da pesquisa Infovis para a Saúde Pública relativos ao painel criado para apoio aos usuários gestores federais do SAMU. A pesquisa teve como foco 
intervenções de design da informação elaboradas com o envolvimento dos usuários. $\mathrm{O}$ artefato originado se constitui como protótipo de um painel operacional para gestão das instalações, do repasse e da cobertura do SAMU em todo o território nacional. Para a elaboração do painel foram empregados diferentes recursos de design gráfico e de visualização de dados, como gráficos de séries temporais, mapas coropléticos, tabelas, big numbers e pictogramas. $\mathrm{O}$ protótipo produzido refletiu diretamente as necessidades identificadas junto ao usuário do Ministério da Saúde, e teve como base de informação o conjunto de dados provenientes do CNES. Abordagens com diferentes usuários, como gestores locais, atendentes ou o cidadão, devem guiar requisitos distintos de visualização do SAMU.

\section{Agradecimento}

Esta pesquisa recebeu apoio financeiro da Organização Pan-Americana da Saúde (OPAS), parte do sistema das Nações Unidas (ONU), como Escritório Regional para as Américas da Organização Mundial da Saúde (OMS) e como a organização de saúde do Sistema Interamericano.

\section{Referências}

Cairo, A. (2013). The Functional Art: An Introduction to Information Graphics and Visualization. New Riders Publishing.

Constituição (1988). Constituição da República Federativa do Brasil. Brasília, DF: Senado Federal: Centro Gráfico.

Gothelf, J., \& Seiden J. (2013). Lean UX: Applying Lean Principles to Improve User Experience. USA: O'Reilly.

Ministério da Saúde. (2012a). Portaria $n^{\circ} 406$, de 8 de março de 2012. Institui a Sala de Apoio à Gestão Estratégica (SAGE) e redefine o fluxo para a gestão das informações estratégicas no âmbito do Ministério da Saúde e entidades a ele vinculadas. Diário Oficial da União. Brasil.

Ministério da Saúde. (2012b). Portaria $n^{\circ}$ 1.010, 21 de maio de 2012. Redefine as diretrizes para a implantação do Serviço de Atendimento Móvel de Urgência (SAMU 192) e sua Central de Regulação das Urgências, componente da Rede de Atenção às Urgências. Ministério da Saúde. Gabinete do Ministro. Brasil.

Paim, J. S. (2009). O que é o SUS. Rio de Janeiro: Editora Fiocruz.

Sanders, E. B.-N., \& Stappers, P. J. (2008). Co-creation and the new landscapes of design. In: CoDesign, 4(1), 5-18. https://doi.org/10.1080/15710880701875068

Sarikaya, A., Correll, M., Bartram, L., Tory, M., \& Fisher, D. (2019). What Do We Talk About When We Talk About Dashboards?. IEEE Transactions on Visualization and Computer Graphics, 25(1), 682-692. https://doi.org/10.1109/TVCG.2018.2864903. 
Schoffelen, J., Claes, S., Huybrechts, L., Martens, S., Chua, A., \& Moere, A. V. (2015). Visualising things. Perspectives on how to make things public through visualisation. CoDesign. 11(3-4), 179-192. https://doi.org/10.1080/15710882.2015.1081240

Simonsen, J., Bærenholdt, J. O., Büscher, M., \& Scheuer, J. D. (2010). Design Research: Synergies from Interdisciplinary Perspectives. London: Routledge. https://doi.org/10.4324/9780203855836

Souza-Junior, M., Alves, A. M., Accioli-Neto, C., Alves, A., \& Souza, H. (2016). Geolocalização aplicada aos atendimentos do SAMU 192 a partir do rastreamento em tempo real das ambulâncias. Anais do XV Congresso Brasileiro de Informática em Saúde, 1011-1018.

Thiollent, M. (2011). Action Research and Participatory Research: An Overview. International Journal of Action Research. International Journal of Action Research, 7(2), 160-174.

Tamaki, E. M., Tanaka, O. Y., Felisberto, E., Alves, C. K. A., Junior, M. D., Bezerra, L. C. A., Calvo, M. C. M, \& Miranda, A. S. (2012). Methodology for construction of a panel of indicators for monitoring and evaluation of unified health system (SUS) management. Ciência \& Saúde Coletiva. 17(4), 839-849. https://doi.org/10.1590/S141381232012000400007

Van der Lugt, R. (2002). Brainsketching and How it Differs from Brainstorming. Creativity and Innovation Management, 11, 43-54. https://doi.org/10.1111/1467-8691.00235

\section{Sobre os autores}

Leandro Manuel Reis Velloso, Dr., USP, Brasil <leandrovelloso@usp.br> Lucia Vilela Leite Filgueiras, Dra., USP, Brasil <lfilguei@usp.br>

Mailson Fontes de Carvalho, Dr., UFPI, Brasil <mailsoncarvalho@ufpi.edu.br> Luiz Sérgio de Souza, Dr., CEETEPS-FATEC, Brasil <luizsds@usp.br> Luciano Anísio Garcia, Me., USP, Brasil <luciano.garcia@usp.br> Johne Marcus Jarske, Me., USP, Brasil <johne.jarske@usp.br> Tânia Letícia dos Santos, Dr., FATEC-SP, Brasil <tania.leticia2011@gmail.com> Gabriela Momberg Araújo, USP, Brasil <gabriela.momberg.araujo@usp.br> Henrique Mathias Fernandes, USP, Brasil <fernandeshm1997@usp.br> Solange Nice Alves de Souza, Dr., USP, Brasil <ssouza@usp.br> Marcia Ito, Dr., CEETEPS-FATEC, Brasil <marciaito2000@gmail.com> Átila Szczecinsky Rodrigues, Ministério da Saúde, Brasil <atila.rodrigues@saude.gov.br> 